\title{
ABUNDANCIA, PREFERENCIA DE HÁBITAT E IMPACTO DEL ECOTURISMO SOBRE EL PUMA Y DOS DE SUS PRESAS EN LA RESERVA DE LA BIÓSFERA EL TRIUNFO, CHIAPAS, MÉXICO
}

\author{
IVÁN LIRA TORRES ${ }^{1}$ Y EDUARDO J. NARANJO PIÑERA²
}

\begin{abstract}
${ }^{1}$ Instituto de Ecología, Universidad del Mar - Campus Puerto Escondido (UMAR). Puerto Escondido, San Pedro Mixtepec, Juquila, Oaxaca, México. C.P. 071980, Tel: (52)954-5883365; Fax: (52) 954-5823550.e-mail: ilira@zicatela.umar.mx

${ }^{2}$ Departamento de Ecología y Sistemática Terrestres, El Colegio de la Frontera Sur (ECOSUR), Ap. 63, San Cristóbal de Las Casas, Chiapas 29290, México. Tel.(52)967-6781883, Fax (52)967-6782322, e-mail: enaranjo@sclc.ecosur.mx
\end{abstract}

\begin{abstract}
Resumen: Los grandes mamíferos tienen un importante papel en la dinámica y estructura de los bosques neotropicales. Sin embargo, su ecología apenas ha sido estudiada en el bosque mesófilo de montaña o nubliselva del sureste de México. En este estudio, evaluamos la abundancia relativa, densidad poblacional, preferencia de hábitat e impacto del ecoturismo del puma (Puma concolor) y dos de sus especies presa; venado temazate (Mazama americana) y pecarí de collar (Tayassu tajacu), a lo largo de 475 km de transectos lineales dentro del Polígono I de la Reserva de la Biosfera El Triunfo, Chiapas, México. Observamos los siguientes índices de abundancia y densidades: M. americana con 0.38 rastros / $\mathrm{km}$ y $0.32 \mathrm{ind} / \mathrm{km}^{2}$, T. tajacu con 0.13 rastros / $\mathrm{km}$ y 1.19 ind / $\mathrm{km}^{2}$ y P. concolor con 0.04 rastros / km. Las tres especies fueron más abundantes en el bosque mesófilo de montaña $(P<0.0001)$ y utilizaron con mayor intensidad el bosque mesófilo de montaña con vegetación secundaria $(P<0.01)$ respecto a otros tipos de vegetación. No se observaron diferencias significativas en los índices obtenidos en cuanto a temporadas seca y lluviosa, entre meses del año, ni entre el uso de senderos con y sin actividad ecoturística para las tres especies. No obstante, se detectó una asociación negativa entre el uso ecoturístico de los senderos con la presencia de M. americana. La degradación del hábitat, parece afectar severamente la permanencia de las tres especies en el área de estudio, por lo que ésta dependerá de evitar, regular y controlar la apertura y tránsito de senderos ecoturísticos para la observación de aves y otras actividades recreativas en el Polígono I de la Reserva de la Biosfera El Triunfo.
\end{abstract}

Abstract: Large mammals have important roles in the dynamics of Neotropical Rainforests.
However, their ecology has barely been studied in the clouded forests of southern Mexico. In
this study, we assessed the abundance, habitat use and impact of ecotourism on populations of
puma (Puma concolor) and two of its prey species: red brocket deer (Mazama americana), and
collared peccary (Tayassu tajacu) along $475 \mathrm{~km}$ of linear transects in the core area of El Triunfo
Biosphere Reserve (REBITRI), Chiapas, Mexico. We observed $0.38,0.13$ and 0.04 tracks/km of
red brocket deer, collared peccary and puma, respectively. Density estimates obtained for the
two ungulate species were 0.32 red brocket deer and 1.19 collared peccaries per square km. The 
three species were more frequently seen in clouded forest $(P<0.01)$ than in other vegetation types. We did not find differences in overall abundance between areas with and without ecotourism activities. However, these activities were negatively correlated with the abundance of red brocket deer in the study area. Our results suggest that recreational use (e.g., birdwatching) should be restricted to a few main trails of REBITRI's core area in order to reduce associated habitat degradation.

Palabras clave: Bosque Mesófilo, Chiapas, Ecología, Mazama americana, Tayassu tajacu, Puma concolor, Reserva de la Biosfera El Triunfo.

\section{INTRODUCCIÓN}

El ecoturismo es una nueva modalidad que busca aprovechar la vida silvestre y ambientes naturales con miras para beneficiar a las poblaciones rurales, con la ventaja de no ser una forma de uso consuntivo. En tanto que el éxito de cosechar animales silvestres puede depender de obtener parámetros fundamentales sobre su abundancia y densidad, el turismo aporta diferentes elementos, que incluyen el efecto de los turistas en comunidades naturales, la repercusión de la cultura y del capital nacional, extranjero y del reto de educar a la gente, mostrándoles los problemas que enfrentan los sistemas naturales (Groom et al., 1997; Piagram, 1980; Touval, 1993).

Un ejemplo de lo anterior, son los actuales programas desarrollados por la Reserva de la Biosfera El Triunfo, en la Sierra Madre de Chiapas, México, donde la exuberante vegetación y la diversidad de vida silvestre neotropical han hecho de esta localidad un área sumamente atractiva para el desarrollo del ecoturismo. Por desgracia, las especies neotropicales que podrían ser interesantes para los turistas como son: tapires, venados, pecaríes, felinos, monos, son especies poco conocidas en el área, con bajas densidades, en algunos casos de hábitos crepusculares y muy esquivas, lo que limita al turista a la observación de aves, como el pavón (Oreophasis derbianus), el quetzal (Pharomachrus mocinno) y la tangara aliazul (Tangara cabanisi). No obstante, aun cuando el ecoturismo ha alentado la valoración económica y conservación de los recursos flora, fauna y de las comunidades asentadas a los alrededores de esta área natural, es probable que esta actividad tenga efectos colaterales adversos sobre la biodiversidad (Groom et al.,1997; INE / SEMARNAP, 1999; Piagram, 1980; Touval, 1993).

Desafortunadamente falta mucha investigación para definir si el ecoturismo es un problema de conservación para la vida silvestre en general y la mastofauna en particular, considerando que esta actividad es una entrada adicional de recursos económicos para el manejo, difusión y conservación de la reserva. El estudio de Lira et al., (2004) sobre ecología del tapir (Tapirus bairdii) en el Polígono I de esta área natural, sugiere que si se siguen abriendo senderos para el desarrollo de actividades 
ecoturísticas es probable que las poblaciones de este ungulado tengan que desplazarse a otras localidades, donde no tendrán la protección adecuada por parte de la reserva y serán más vulnerables a la cacería, como se observó en los límites de varias comunidades asentadas en esta región. No obstante, existe un abismo de información para determinar si lo encontrado en dicho estudio es aplicable a otros mamíferos locales. El presente estudio involucra a una de las Regiones Terrestres Prioritarias del sureste de México (RTP 133: El Triunfo - La Encrucijada - Palo Blanco) y continua con un programa de monitoreo en la región central de la Sierra Madre de Chiapas, específicamente en la zona núcleo y de amortiguamiento del Polígono I de la Reserva de la Biosfera El Triunfo. Esta área natural protegida cuenta con poblaciones importantes de venado temazate (Mazama americana), pecarí de collar (Tayassu tajacu) y puma (Puma concolor) (Espinoza et al., 1998), aún cuando no existen estudios específicos que apoyen esta suposición, por lo que en este estudio nos enfocamos en estimar la abundancia relativa, densidad y preferencia hábitat del $M$. americana, T. tajacu y P. concolor en el Polígono I de la Reserva de la Biosfera El Triunfo y su posible correlación negativa con el ecoturismo.

\section{MÉTODOS}

\section{Área de Estudio}

La Reserva de la Biosfera El Triunfo (REBITRI), creada por decreto presidencial en marzo de 1990. Esta localizada en la región central de la Sierra Madre de Chiapas, entre

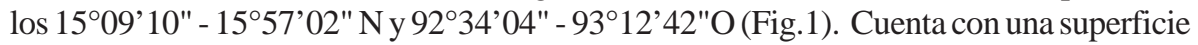
de 119,177 ha, dividida en dos zonas de manejo: a) la zona núcleo, conformada por cinco polígonos (I El Triunfo, II Ovando, III Cuxtepeques, IV El Venado, y V La Angostura), destinada a la protección e investigación de la biodiversidad y a la educación ambiental (25,763 ha); y b) la zona de amortiguamiento, con un solo polígono que envuelve a las zonas núcleo (93,458 ha), destinada a promover el desarrollo de actividades productivas sustentables. El Polígono I (El Triunfo), es el más accesible y grande de todos, abarca una extensión de 10,000 ha, con rangos altitudinales que van desde los 1000 a los 2450 msnm. Los climas predominantes según la clasificación de Köppen; modificado por García (1973), son el semicálido húmedo y el templado-húmedo con abundantes lluvias en verano. La temperatura media anual del área fluctúa entre $18-22^{\circ} \mathrm{C}$ y la precipitación anual es de 2500 - $3500 \mathrm{~mm}$ (Arreola et al., 1997; Heath and Long, 1991; Long y Heath, 1991; INE/SEMARNAP 1999; Lira et al., 2004). Se han descrito siete comunidades vegetales para el área: Gaultheria-UgniVaccinium, Quercus-Matudaea-Hedyosmun-Dendropanax, Liquidambar-QuercusPinus, Cupressus-Pinus, Ficus-Coccoloba-Dipholis-Sapium, Garcinia-Inga- 
Desmopsis y Quercus salicifolia, todas estas enmarcadas en el sistema de clasificación de Breedlove (1981), Long - Heath (1991) y Rzedowski (1991) de Bosques Mesófilos de Montaña.

\section{Selección de Sitios de Muestreo}

El presente estudio se llevó a cabo de febrero a diciembre del 2001 en el Polígono I de la REBITRI, consistiendo en un trabajo de campo de 11 meses con salidas entre ocho y nueve días por mes, seis en época seca y cinco en época de lluvias. Del total de transectos $(N=12)$, seis fueron seleccionados en veredas amplias ( $2 \mathrm{~m}$ de ancho como mínimo), previamente establecidas por personal de la reserva y utilizadas frecuentemente en actividades ecoturísticas (observación de aves, excursionismo, visitas escolares, etc.). Los otros seis se ubicaron en senderos cerrados al turismo y veredas que la fauna silvestre utiliza para desplazarse (transectos lineales de amplitud variable). La longitud de cada uno de los transectos varió entre 0.50 a $10 \mathrm{~km}$, procurando, en lo posible, que cada transecto se ubicara dentro de un solo tipo de hábitat según el Inventario Nacional Forestal del año 2000 para esta área: bosque mesófilo de montaña, bosque mesófilo de montaña con vegetación secundaria y cafetal tecnificado Lira et al., (2004) (Fig.1).

\section{Abundancia Relativa}

Se realizaron conteos de rastros identificables tales como huellas y excretas para estimar la abundancia relativa de M. americana, $T$. tajacu y $P$. concolor en el área de estudio, apoyándose en las guías de campo de Aranda y March (1987) y Aranda (1994 y 2000). El criterio que se utilizó para la identificación de huellas fue la estructura anatómica, mientras que en el caso de las excretas se consideró forma, olor, tamaño, textura y asociación con huellas en el caso de $P$. concolor. Los conteos se realizaron entre las 08:30-17:00 h y el tiempo mínimo entre dos conteos en un mismo transecto fue de 20 días. Para evitar una sobreestimación de la abundancia, se contaron como distintos a grupos de rastros que tuvieran como mínimo una separación de 100 m entre sí. Los índices de abundancia relativa (AR) se calcularon con la siguiente fórmula: AR $=N r / a ̊ k m($ Naranjo, 2000).

Donde: $\mathrm{Nr}=$ numero de rastros observados.

åkm = Suma de kilómetros recorridos.

Estos índices fueron calculados por cada transecto, mes y rango altitudinal. Debido a que los índices no se ajustaron a una distribución normal, se hicieron 

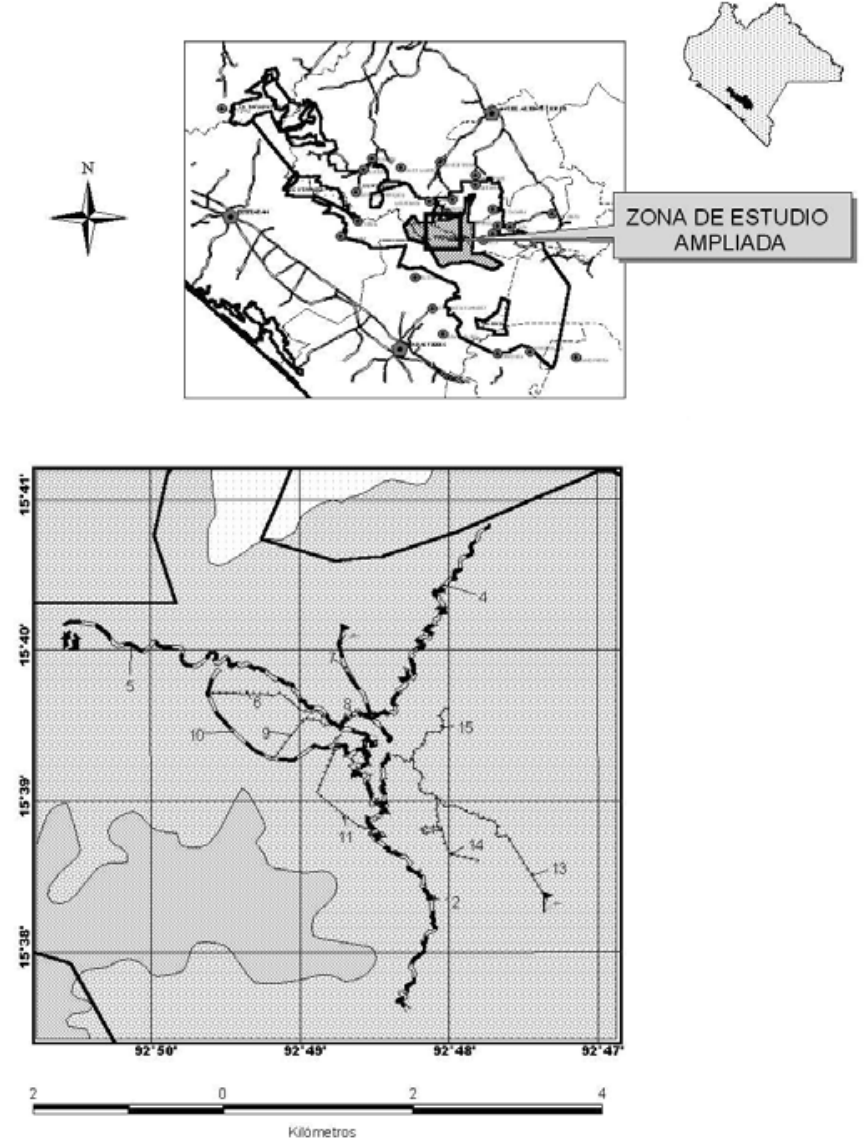

Figura 1. Localización del área de estudio, tipos de vegetación de acuerdo al Inventario Nac. Forestal 2000 y abreviaturas de transectos recorridos en la Reserva de la Biosfera el Triunfo (Polígono I), Chiapas, México. Transectos c / Ecoturismo: S. Palo Gordo = 5, Camino al Triunfo $=4$, S. Interpretativo $=8$, C. Triunfo $=7$, S. Viejo $=10$, S. Costa $=12$. Transectos s / Ecoturismo: S. Filo Quetzal = 6, S. Raicero $=9$, S. Mono $=11$, S. Cerro Bandera $=13$, S. Teja $=14$, S. Palmar $=15$. 
comparaciones por medio de una prueba de Kruskal-Wallis. Las variables como estación (seca y lluviosa), vegetación (bosque mesófilo y vegetación secundaria) e impacto humano (ecoturismo y sin ecoturismo) fueron analizadas mediante la prueba Suma de Rangos de Mann-Whitney (Sokal y Rohlf 1995). Todos los análisis estadísticos se realizaron con el programa Sigma stat 1.0 con un nivel de significancia del 95\% (Jandel Corp., 1993).

\section{Densidad Poblacional}

Se hicieron observaciones directas, anotando el número de animales observados respecto a la distancia perpendicular y longitud del transecto. La densidad se estimó mediante el método de Buckland et al., (1993), utilizando el programa DISTANCE 3.5 (Thomas et al., 1998).

$D=\mathrm{nA} * \mathrm{f}(0) / 2 \mathrm{~L}$ ( $N$ de individuos $/ \mathrm{km}^{2}$; Buckland et al., 1993).

Donde: $D=$ densidad

$\mathrm{n}=\mathrm{n}^{\circ}$ de individuos observados

$\mathrm{A}=$ área de muestreo $\left(\mathrm{km}^{2}\right)$

$\mathrm{L}=$ longitud del transecto $(\mathrm{km})$

$\mathrm{f}(0)=$ probabilidad de detección calculada a partir de las distancias perpendiculares de observación.

\section{Preferencia de Hábitat}

A partir de las cartas geográficas generadas en el Inventario Nacional Forestal 2000 y bases de datos de la Dirección General de la Reserva de la Biosfera El Triunfo y de El Colegio de la Frontera Sur (ECOSUR), se construyeron mapas de la zona de estudio a escala gráfica de 1:479190, 1:81813 y de cobertura vegetal a escala gráfica de 1:70131 en el Laboratorio de Análisis Geográficos del Instituto de Historia Natural y Ecología (IHNE). La preferencia de hábitat se estimó mediante la prueba de Chi-cuadrada, por medio del número de huellas y excretas se obtuvo la frecuencia observada, esperada y los intervalos de Bonferroni para cada tipo de cobertura vegetal utilizada por las especies, empleando el programa HABUSE 4.0 (Byers et al., 1984).

\section{RESULTADOS}

Se recorrieron $475 \mathrm{~km}$ de transectos lineales, observando un total 172 rastros (huellas y excretas) de las tres especies. Del total, 101 (58.7\%) correspondieron a M. americana, $37(21.5 \%)$ a $T$. tajacu y $34(19.8 \%)$ a $P$. concolor. El índice de abundancia relativa promedio en el área de estudio fue de $0.38 \pm 0.49$ (D.E.) rastros / $\mathrm{km}$ para M. americana; 
vol. 7
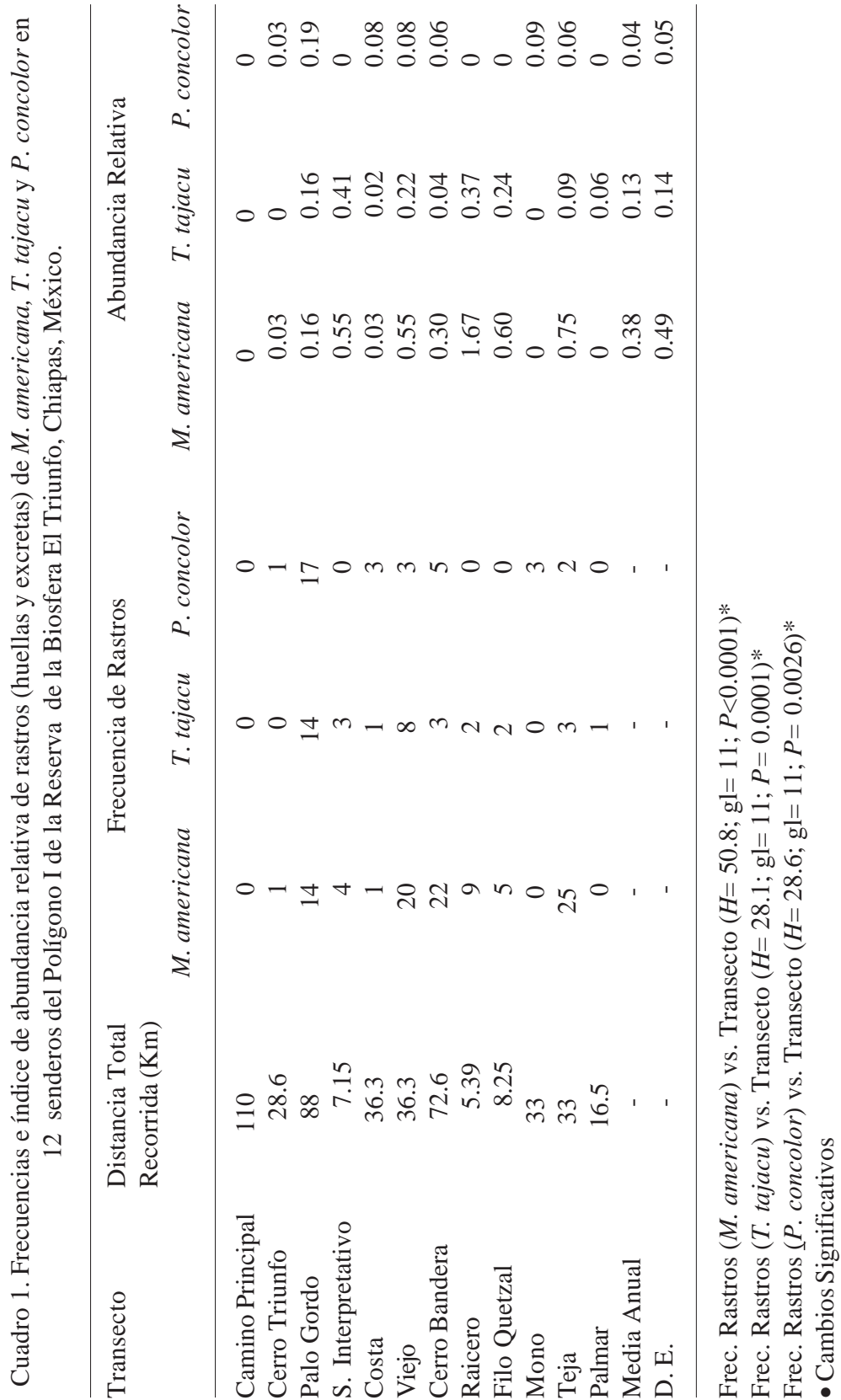
$0.13 \pm 0.14$ rastros / km para T. tajacu y $0.04 \pm 0.05$ rastros / km para $P$. concolor. Los transectos con mayor índice de abundancia de rastros para $M$. americana fueron Raicero con 1.67 / km, Filo Quetzal con 0.60 / km y Teja con 0.75 / km ( $H=50.8$; gl= 1; $P$ $<0.0001$ ); para T. tajacu fueron S. Interpretativo con 0.41 / km, Raicero con 0.37 / km y Filo Quetzal con 0.24 / km ( $H=28.1$; gl= 11; $P<0.0001)$, y para $P$. concolor fueron Palo Gordo con 0.19 / km, Mono con 0.09 / km, Costa y Viejo con 0.08 / km respectivamente $(H=28.6 ; \mathrm{gl}=11 ; P<0.0025)$ (Cuadro 1$)$.

Comparando los índices de abundancia relativa obtenidos por tipo de vegetación, se obtuvieron mayores valores en el bosque mesófilo de montaña respecto a la vegetación secundaria para $M$. americana $(U=2289.5 ; \mathrm{gl}=1 ; P<0.0001), T$. tajacu $(U=944.0 ; \mathrm{gl}=1 ; P<0.0001)$ y $P$. concolor $(U=902.0 ; \mathrm{gl}=1 ; P<0.0001)$ (Cuadro 2). No se observaron diferencias en los índices reportados en cuanto a temporadas seca y lluviosa: $M$. americana $(U=3738.0 ; \mathrm{gl}=1 ; P=0.2499)$, T. tajacu ( $U=4166.0$; gl= $1 ; P=0.3415)$ y $P$. concolor $(U=4229.5$; gl= $1 ; P=0.2736)$; ni entre meses del año: $M$. americana $(H=5.77 \mathrm{gl}=10 ; P=0.8346)$, . tajacu $(H=15.0 \mathrm{gl}=10$; $P=0.1305)$ y $P$. concolor $(H=13.6 \mathrm{gl}=10 ; P=0.1914)$; ni entre el uso de veredas naturales y transectos con actividad ecoturística: $M$. americana $(U=4251.5$; gl= $1 ; P=$ 0.5325), T. Tаjаси ( $U=4462.0$; gl= $1 ; P=0.4289)$ y $P$. concolor $(U=4589.5$; gl= 1 ; $P=0.3616)$. El rango altitudinal donde se observaron las mayores frecuencias de rastros para las tres especies fue entre los 1800 y 2100 msnm (Cuadro 3 y 4).

Durante los recorridos realizados sólo se logró la observación directa de seis individuos de $M$. americana y 17 de T. tajacu. La densidad dentro del Polígono I de M. americana y T. tajacu (+ error estándar) estimada en $475.1 \mathrm{~km}$ recorridos fue de 0.32 $\pm 0.20 \mathrm{ind} / \mathrm{km}^{2}$ y $1.19 \pm 0.85 \mathrm{ind} / \mathrm{km}^{2}$ respectivamente (Cuadro 5).

De acuerdo a los rastros obtenidos (huellas y excretas), el bosque mesófilo de montaña con vegetación secundaria fue el hábitat preferido por $M$. americana $\left(X^{2}=\right.$ 121.8; gl= 3; $P<0.01)$, T. tajacu $\left(X^{2}=4.155\right.$; gl= $\left.3 ; P=0.1230\right)$ y $P$. concolor $\left(X^{2}=13.961\right.$; $\mathrm{gl}=3 ; P=0.0013)$, mientras que el bosque mesófilo primario, no obstante de su extensión, le siguió en cuanto a utilización por las tres especies (Cuadro 6).

\section{DISCUSIÓN}

Los mayores índices de abundancia relativa correspondieron a $M$. americana $(0.38$ rastros / km) en transectos localizados en el bosque mesófilo de montaña y alejados de las zonas más perturbadas. Esto posiblemente se deba a la disponibilidad de cuerpos de agua, abundante follaje verde, densa cobertura vegetal y baja presión de cacería que estas áreas puede brindar. Por otro lado, no se observaron diferencias estaciónales ni mensuales en las frecuencias de rastros de $M$. americana, debido posiblemente a condiciones similares del ambiente y terreno, lo que propicia buenas condiciones para la impresión de huellas (Cuarón et al., 2001). 
vol. 7

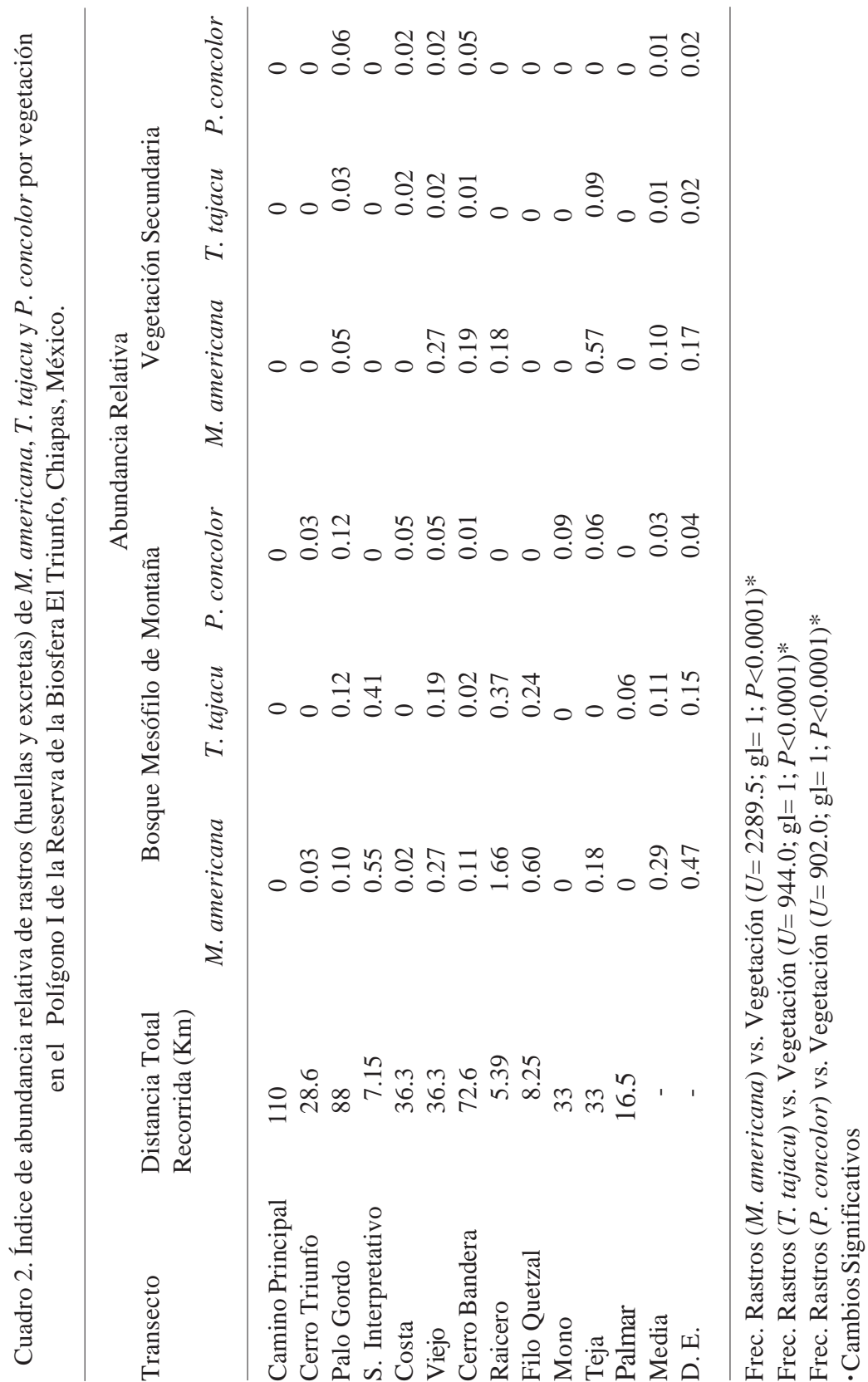




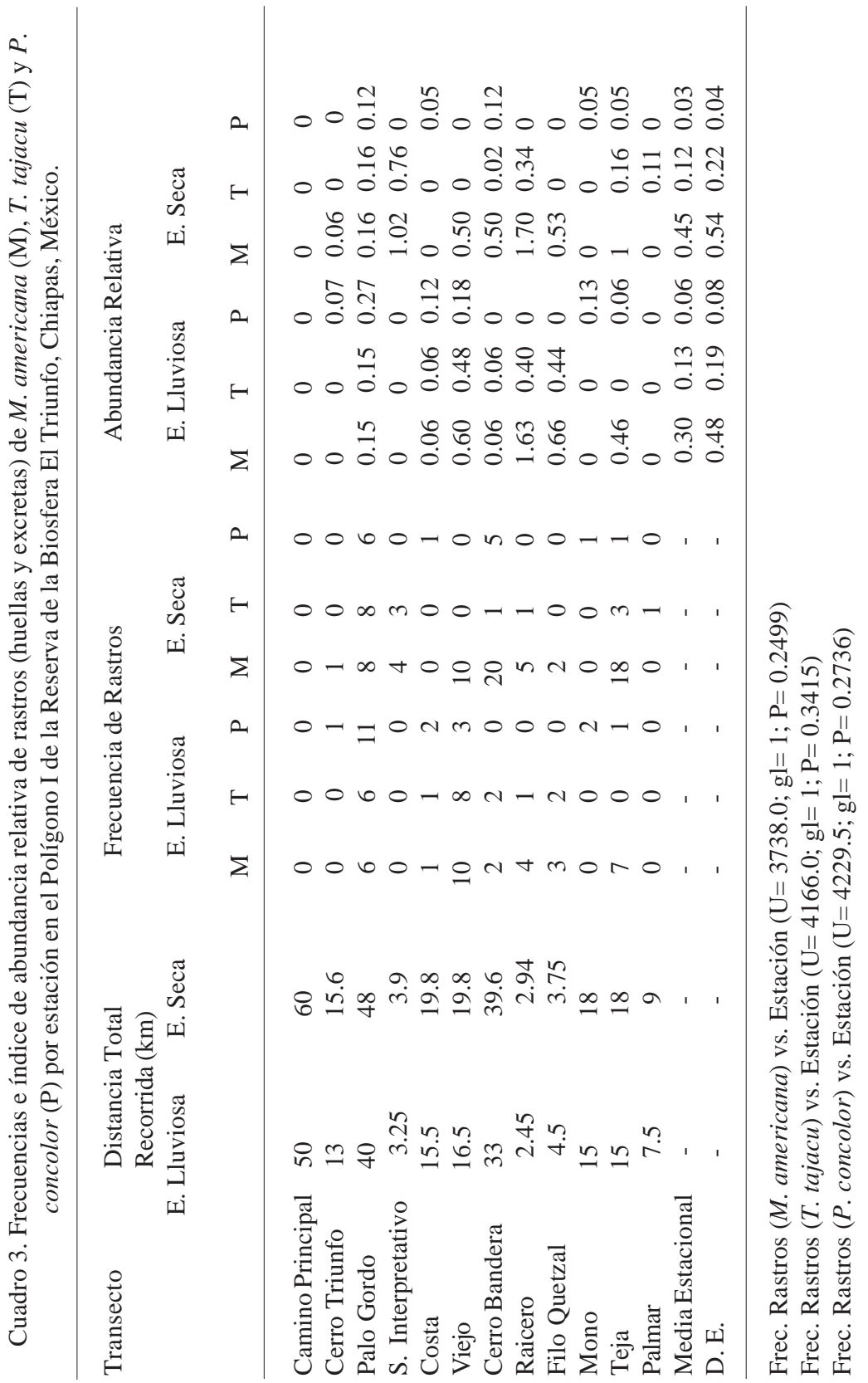


vol. 7

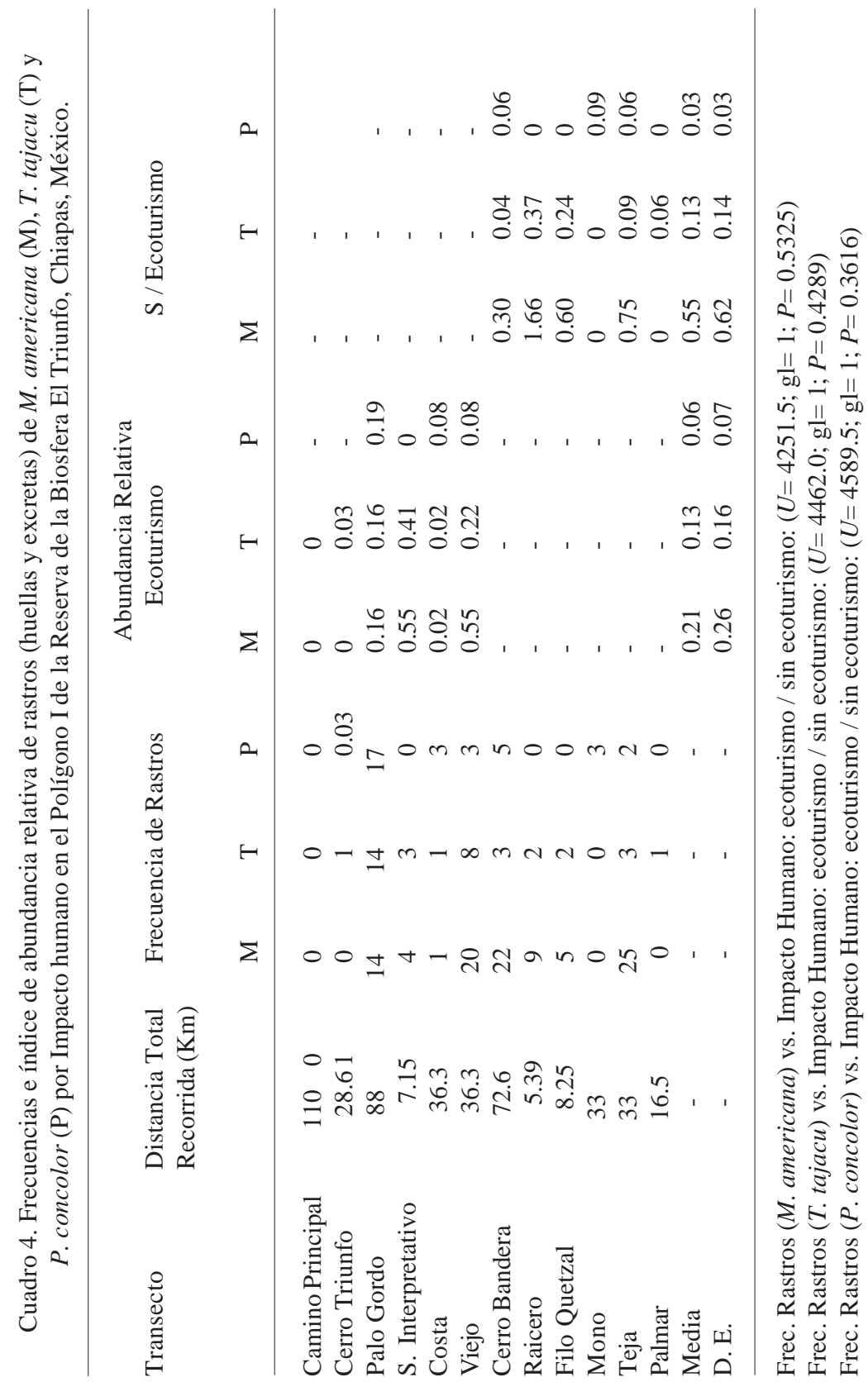




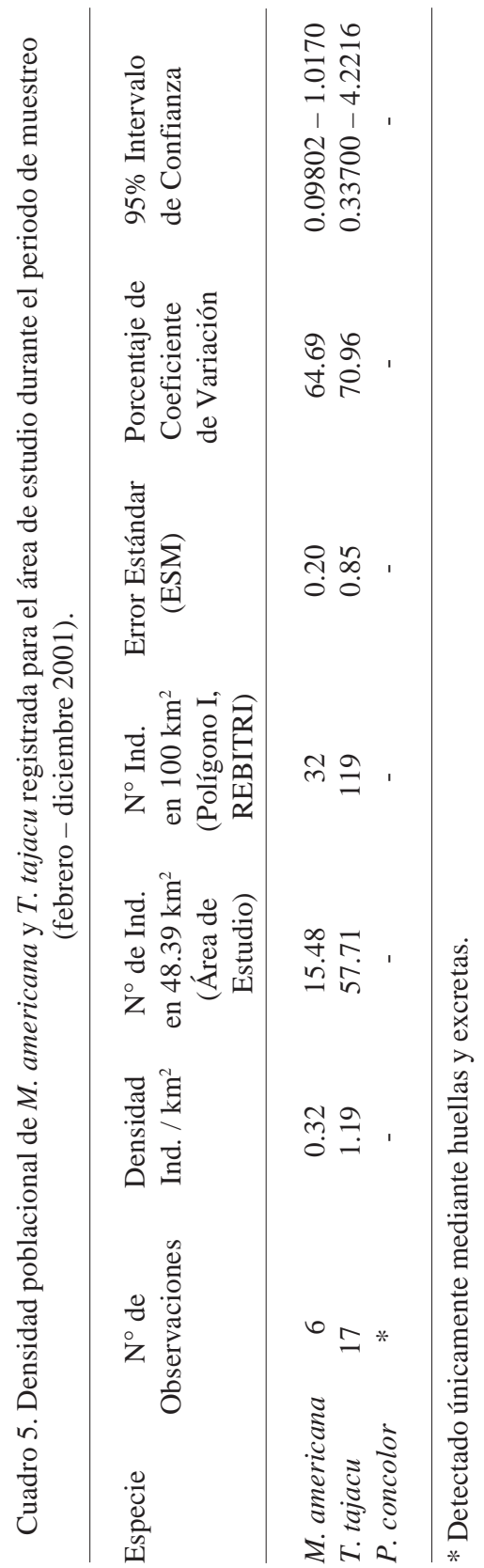




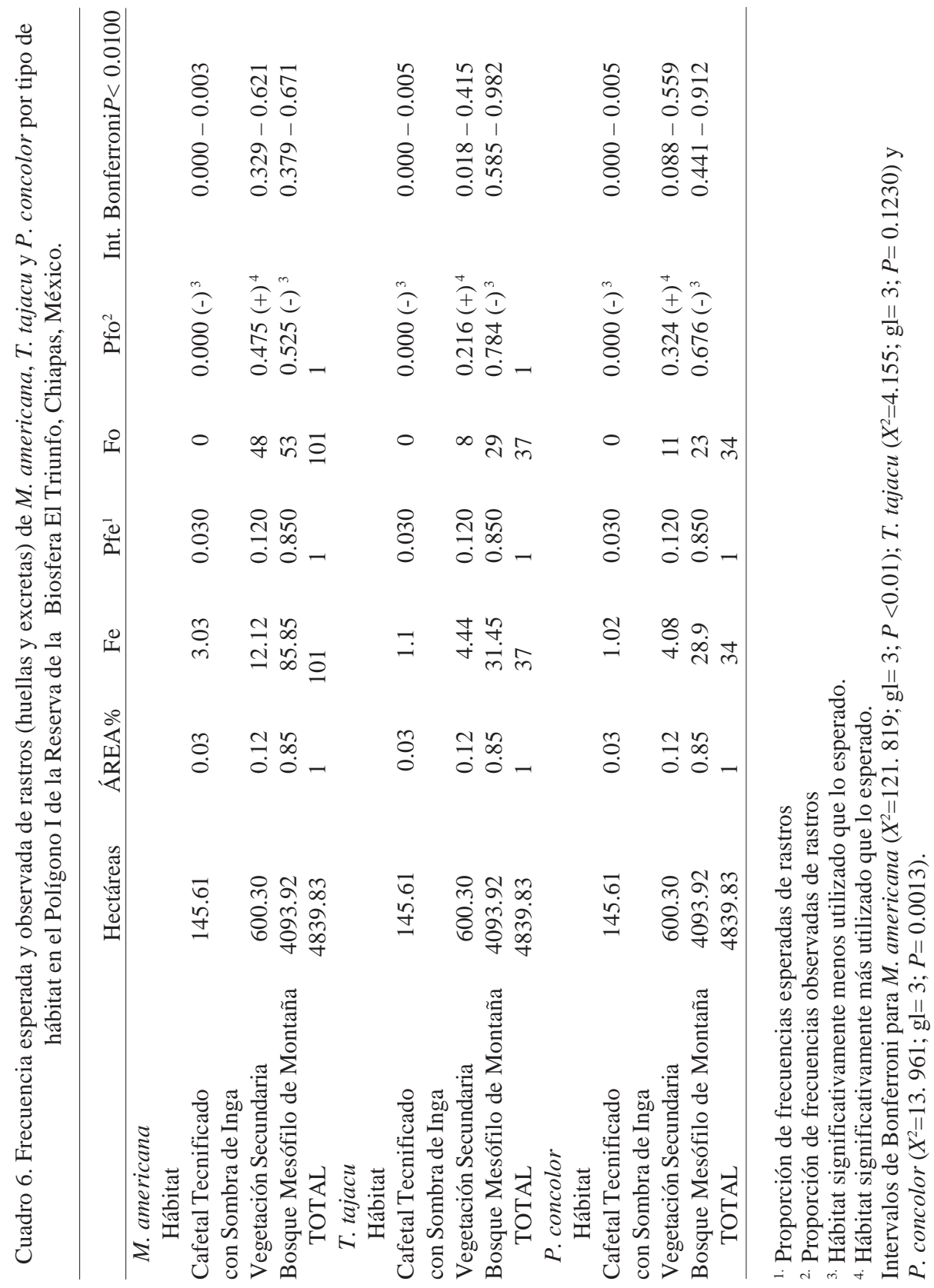


Pese a que no se percibieron diferencias significativas en la abundancia de M. americana por impacto del ecoturismo, marcados contrastes en los índices obtenidos reflejan un mayor uso de veredas naturales respecto a los senderos establecidos por personal de la reserva, percibiéndose una conducta muy similar a la reportada por Noss y Cuéllar (2000) para los ungulados del Chaco Boliviano. El venado temazate en nuestra área de estudio cruzaba los caminos preestablecidos, evitando hacer recorridos largos sobre ellos y registrándose una mayor actividad en las partes terminales donde la frecuencia de tránsito es mínimo.

Sin embargo, si se siguen abriendo senderos para el desarrollo de actividades ecoturísticas es probable que las poblaciones de este ungulado tengan que desplazarse a otras localidades, donde no tendrán la protección adecuada por parte de la reserva y serán más vulnerables a la cacería, como lo observado por Lira et al., (2004) para T. bairdii, en los límites de varias comunidades asentadas en la región y lo expuesto por Groom (1990) y Groom et al., (1997), quienes observaron una reducción de la fauna silvestre amazónica; con excepción de las aves que habitan la playa, alrededor de los centros vinculados al turismo en el Parque Nacional Manu, Perú.

Comparativamente, trabajos llevados a cabo en Quintana Roo, México, por Merediz (1995) y Quijano (1988) con M. americana, mostraron abundancias relativas más bajas que en el presente estudio (0.22 y 0.15 rastros / km respectivamente); así como lo reportado por Bolaños (2000) (0.30 rastros / km) para la cuenca del río Lacantún y Naranjo (2002) (0.11 rastros / km) para la selva lacandona, Chiapas, México, atribuibles a factores tales como una menor tolerancia a las actividades humanas como la cacería y la fragmentación del hábitat. No obstante, Bello y Mandujano (1992) en los Tuxtlas, Veracruz, México, calcularon índices más altos que los obtenidos en el presente trabajo ( 0.79 rastros / $\mathrm{km}$ ), diferencia que tal vez pueden explicarse considerando: a) la inclusión en el trabajo de otros rastros distintos a las huellas y excretas (echaderos y comederos) para la estimación de abundancia; b) diferencias en la implementación de métodos y c) extinción de depredadores potenciales, tal es el caso del Jaguar (Panthera onca) y el Puma (P. concolor) en los Tuxtlas (Coates - Estrada y Estrada, 1986).

En el caso de T. tajacu, que es la segunda especie con el valor más alto de abundancia relativa ( 0.13 rastros / km), encontramos que el pecarí de collar tiene una asombrosa habilidad de adaptarse ha hábitat particularmente fragmentados y con moderadas presiones de cacería, como lo refiere Bodmer y Sowls (1996), Fragoso (1988), March (1990) y Naranjo (2002), y posiblemente eso refleje el que no existan diferencias significativas en cuanto a la utilización de senderos construidos para el desarrollo del ecoturismo y las veredas naturales, así como también a la inexistencia de diferencias estacionales, mensuales en las frecuencias de rastros, debido a condiciones similares del ambiente y terreno. Por otro lado, la abundancia obtenida es mucho menor a la registrada en otros trabajos realizados en México; Merediz (1995) reportó 
índices de 0.21 rastros / km en la selva mediana subperennifolia de Quinta Roo, Quijano (1988) en Tres Reyes, Quintana Roo, obtuvo 0.47 rastros / km, en tanto que Bello y Mandujano (1992) encontraron una abundancia de 0.45 rastros / km en los Tuxtlas, Veracruz, finalmente, Bolaños (2000) obtiene una abundancia relativa de 0.55 rastros / km en la Cuenca del río Lacantún y Naranjo (2002) obtiene una abundancia relativa de 0.28 rastros / $\mathrm{km}$ en la Selva Lacandona. Es de esperarse que, al aumentar el esfuerzo de muestreo $(>475.1 \mathrm{~km})$, se incremente el número acumulado de observaciones de rastros de T. tajacu.

Respecto a la baja abundancia relativa de $P$. concolor ( 0.04 rastros / km), era de esperase, dado que la observación de felinos en libertad comúnmente es un evento poco frecuente, de manera que el registro de sus rastros (huellas y excretas) se ha utilizado en diversas ocasiones para su estudio en el campo (Emmons, 1987). Además por su posición en la cima de la pirámide trófica, este depredador es naturalmente poco abundante. Otro punto importante para considerar, es el papel que juega el puma en la dinámica de los ecosistemas, influyendo en la evolución de las especies presa y constituyendo un factor regulador del incremento de sus poblaciones (Schaller, 1967). Si desaparecieran los pumas del Polígono I de la REBITRI, las poblaciones de venado temazate tenderían a incrementarse y posiblemente los individuos competirían más intensamente entre sí, provocando una posible degradación del hábitat. En este sentido, es importante mencionar que el venado temazate tiene una gran capacidad cursorial por lo que son cazados principalmente por pumas. Esta idea es apoyada por el hecho de que en el 100\% de las 28 excretas de puma recolectadas en esta localidad se encontraron restos (pelos, huesos, cascos) de M. americana, evidenciado la frecuencia de uso y búsqueda de esta especie por parte del depredador. A este respecto, Aranda (1994) comenta que no se conoce un lugar donde el puma prospere si no existe alguna especie de venado, y en Calakmul, México, las presas principales del puma son los venados. Ceballos y Miranda (1986) por su parte comentan que la única restricción que tiene el puma para ocupar una zona determinada es la ausencia de su presa principal: el venado; sin embargo, también su dieta incluye ocasionalmente armadillo, pecarí de collar, puerco espín, aves y reptiles.

Es posible que a diferencia de $M$. americana, el puma se vea beneficiado con la apertura de estos senderos ecoturísticos, lo cual reportaron Noss y Cuéllar (2000) para los carnívoros del Chaco Boliviano y fue corroborado en esta investigación. Este carnívoro, a diferencia de $P$. onca, tiene una mayor adaptabilidad y tolerancia a los climas extremosos de la región, recorriendo frecuentemente áreas abiertas, donde muy posiblemente puede emboscar más fácilmente a sus presas potenciales y tener una mayor maniobrabilidad que en áreas con sotobosque sumamente denso. La abundancia obtenida en este trabajo es mucho mayor a la registrada por Naranjo (2002) con 0.005 rastros / km en la Selva Lacandona, Chiapas, reflejando que entre uno de los principales factores que puedan favorecer la abundancia de esta especie en 
el Polígono I de la REBITRI, sean una menor densidad y abundancia de jaguares en toda la Sierra Madre de Chiapas (Aranda, 1996).

La densidad obtenida en este estudio para M. americana y T. tajacu es baja dentro de los rangos estimados en otras localidades con baja presión de cacería (Alonso, 1997; Bodmer, 1997; Bodmer, Aquino y Navarro, 2000; Bolaños, 2000; Branan y Marchinton, 1987; Glanz,1982; Mandujano, 1991; Naranjo, 2002; Polisar et al.,1998; Quijano, 1988; Quinto, 1994; Townsend, 1996; Wallace y Painter, 2000), sin embargo, es necesario considerar que nuestras estimaciones se basaron exclusivamente en recorridos diurnos, cuando estos animales muestran poca actividad. No obstante, existe un marcado contraste entre este resultado con respecto a la abundancia relativa de estas dos especies para esta misma área; la cual esta dentro de los rangos estimados para otras regiones neotropicales, siendo un indicio de que ambas especies se encuentran en buen estado de conservación dentro del Polígono I de la REBITRI.

Considerando los rastros obtenidos (huellas y excretas), el bosque mesófilo de montaña con vegetación secundaria fue el hábitat significativamente más utilizado que lo esperado por M. americana, T. tajacu y P. concolor. Este hábitat tiene gran importancia dentro del área de estudio ya que no ha sido fragmentado por la actividad humana, sino que, por el contrario, es producto de la heterogeneidad provocada por las fuertes lluvias y deslaves de 1998 en la región, los cuales afectaron fuertemente la costa de Chiapas. El estado de sucesión secundaria en este hábitat es grande, existiendo una gran cantidad de rebrotes y plántulas de árboles, arbustos y hierbas sumamente atractivos para los venados y pecaríes, e ideal para que el puma los aceche. La preferencia del hábitat aquí expuesto coincide en cuanto a que la disponibilidad de alimento y agua son factores importantes en la selección de hábitat por las especies (Galindo y Weber, 1998).

\section{AGRADECIMIENTOS}

Los autores agradecen al Consejo Nacional de Ciencia y Tecnología (CONACYT), por la beca otorgada para realizar estudios de Posgrado (registro 153027), así como al Colegio de la Frontera Sur (ECOSUR) e Idea Wild, por el financiamiento otorgado y equipo de campo donado. También agradecemos de manera muy especial la asesoria brindada por el Biólogo Benjamín Gastón Gordillo Gómez, en el uso de los Sistemas de Información Geográficos del I.H.N.E y a la Dirección General de la Reserva de la Biosfera El Triunfo por las facilidades prestadas para la realización de este estudio.

\section{LITERATURACITADA}

Alonso A. J. 1997. Estado Actual de la Fauna Silvestre en la Propuesta Reserva Comunal del Pucacuro. Pp.93-103. en: Manejo de Fauna Silvestre en la Amazonía. (Fang T. G., R. E. Bodmer, R. Aquino, y M. Valqui, eds.) Instituto de Ecología, La Paz, Bolivia. 
Aranda, M. \& I. March. 1987. Guía de los Mamíferos Silvestres de Chiapas. Instituto Nacional deInvestigaciones sobre Recursos Bióticos, Xalapa, Veracruz.

Aranda, M. 1994. Diferencias entre huellas de jaguar y puma: un análisis de criterios. Acta Zoológica Mexicana (nueva serie), 63:75-78.

Aranda, M. 1994. Los pecaríes en la alimentación del jaguar. Acta Zoológica Mexicana (nueva serie), 62:11-22.

Aranda, M. 1996. Distribución y abundancia del jaguar, Panthera onca (Carnívora; Felidae) en el Estado de Chiapas, México. Acta Zoológica Mexicana (nueva serie), 68:45-52.

Aranda, M. 2000. Huellas y otros rastros de los mamíferos grandes y medianos de México. Instituto de Ecología A.C., Xalapa, Veracruz.

Arreola, M. A., L. C. Noble, M. A. Altamirano, M. E. Vergara, L. F. G. Zúñiga, R. A. Becerril, G. G. Cuevas y C. V. Guillén. 1997. Evaluación de los cambios de uso del suelo y la cobertura vegetal en la Reserva de la Biosfera El Triunfo, Chiapas, México. Instituto para el Desarrollo Sustentable en Mesoamérica, A. C., WWF, IHN, INE. San Cristóbal de Las Casas, Chiapas.

Arriaga, L., J. M. Espinoza, C. Aguilar, E. Martínez, L. Gómez y E. Loa (coordinadores). 2000. Regiones terrestres prioritarias de México. Comisión Nacional para el Conocimiento y Uso de la Biodiversidad, México D.F.

Bello, J. y S. Mandujano. 1992. Distribución y abundancia relativa de las especies del órden Artíodactila en Los Tuxtlas, Veracruz. Pp. 199 - 211, en: Memorias del X Simposio sobre Fauna Silvestre. Facultad de Medicina Veterinaria y Zootecnia, UNAM, México D.F.

Bodmer, R. E. y K. L. Sowls. 1996. El pecarí de collar. Pp. 5-15, en: Plan de Acción y Evaluación de la Condición actual de los Pecaríes (W. Oliver, ed.) IUCN, Gland, Suiza.

Bodmer, R. E., R. Aquino y P. Puertas. 1997. Alternativas de Manejo para la Reserva Nacional Pacaya -Samiria: Un Análisis sobre el Uso Sostenible de la Caza. Pp. 65-74, en: Manejo de Fauna Silvestre en la Amazonía.(T. G. Fang, R. E. Bodmer, R. Aquino y M. Valqui, eds.), Instituto de Ecología, La Paz, Bolivia.

Bodmer, R. E., R. Aquino, P. Puertas, C. Reyes, T. G. Fang, y N. Gottdenker. 1997. Manejo y Uso Sustentable de Pecaríes en la Amazonía Peruana. Ocasional Paper of the IUCN Species comisión $N^{\circ} 18$ IUCN- Sur, Quito, Ecuador.

Bodmer, R. E., R. Aquino y J. G. G. Navarro. 2000. Sustentabilidad de la caza de mamíferos en la Cuenca del Río Samiria, Amazonía Peruana. Pp.447-469, en: Manejo de Fauna Silvestre en Amazonía y Latinoamérica. Asunción, Paraguay. (Cabrera, E. C. Mercolli, y R. Resquin, eds), Instituto de Ecología, La Paz, Bolivia.

Bolaños, J. E. 2000. Densidad, abundancia relativa, distribución y uso local de los ungulados en la Cuenca del Río Lacantún, Chiapas, México. Tesis de Licenciatura. Universidad de Ciencias y Artes del Estado de Chiapas. Escuela de Biología, Tuxtla Gutiérrez, Chiapas.

Brannan, W.V. y R. L. Marchinton. 1987. Reproductive ecology of white-tailed and red brocket deer in Suriname Pp. 344 - 351, en: Biology and Management of the Cervidae. (C. M. Wemmer, ed.). Smithsonian Institution Press, Washington, D.C.

Breedlove, D. E. 1981. Flora of Chiapas. Part 1. Introduction to the Flora of Chiapas. California Academyof Sciences, San Francisco, California. 
Buckland, S. T., D. R. Anderson, K.P. Burnham, y J.L. Laake. 1993. Distance sampling: estimating abundance of biological populations. Chapman \& Hall, London, UK.

Byers, C. R., R. K. Steinhorst y P.R. Krausman. 1984. Clarification of a technique for analysis of utilization-availability data. Journal of Wildlife Management, 48:1050-1053.

Ceballos, G. y A. Miranda. 1986. Los Mamíferos de Chamela, Jalisco. Universidad Nacional Autónoma deMéxico, Instituto de Biología, México, D. F. 436 pp.

Coates - Estrada, R. y A. Estrada. 1986. Manual de Identificación de Campo de los Mamíferos de la Estación de Biología “Los Tuxtlas”. Instituto de Biología. Universidad Nacional Autónoma de México, México D.F.

Cuarón, D. A., G. T. García, C. Gonzáles-Baca, A. M. D. González, A. B. CH. López, y P. Cartón. 2001. How long does a tapir track last? Implications for population assessments. First International Tapir Symposium, San José, Costa Rica.

Emmons, L. H. 1987. Comparative feeding ecology of felids in a neotropical forest. Behavior Ecology Sociobiology, 20:271-283.

Espinoza, M. E., A. Anzures, y E. Cruz. 1998. Los Mamíferos de El Triunfo. Revista Mexicana de Mastozoología, 3:79-94.

Fragoso, J. M. 1988. Home range and movement patterns of white-lipped peccary (Tayassu pecari) herds in the norther Brazilian Amazon. Biotropica, 30: 458 - 469.

Galindo - Leal, C., y M. Weber. 1998. El Venado de la Sierra Madre Occidental. EDICUSA CONABIO, México D.F.

García, E. 1973. Modificaciones al sistema de clasificación climática de Köppen. Instituto de Geografía, U.N.A.M. México D.F.

Glanz, W. E. 1982. The terrestrial mammal fauna of Barro Colorado Island: censuses and long - term changes. Pp. 455 - 468, en: The Ecology of a Tropical Forest: Seasonal Rhythms and Long - Term Changes. (E.G. Leigh, A. S. Rand y D. M. Windsor, eds.) Smithsonian Institution Press, Washington, D.C.

Groom, M. J. 1990. Management of ecotourim in Manu National Park: Controlling negative effects on beach nestin birds and animals. Procedings of First International Symposium on Ecotourism andResource Conservation. (J. Kuslev y J. Andrews, comps.) Association of Wetland Managers, Washington, D.C.

Groom, M. J., R. D. Podolsky y C. A. Munn. 1997. El Turismo como aprovechamiento sostenido de la vida silvestre: un caso práctico en Madre de Dios, en el Sureste de Perú. Pp. 459 - 482, en: Uso y Conservación de la Vida Silvestre Neotropical (Robinson J. G. y K. H. Redford, eds.). Smithsonian Institution Press, Washington, D.C.

Heath, M. and Long, A. 1991. Habitat, distribution and status of the Azure-rumped Tanager Tangara cabanisi in México. Bird Conservation International,1: 223-254.

INE / SEMARNAP. 1999. Programa de Manejo de la Reserva de La Biosfera El Triunfo, México. INE -SEMARNAP. México D.F.

Inventario Nacional Forestal. 2000. Escala 1:250,000.SEMARNAT.

Jandel Coorporation. 1993. Sigma Stat for Windows Vers. 1.0. San Rafael, California.

Lira , I., P. E. Naranjo, A. D. Güiris y A. E. Cruz. 2004. Ecología del Tapirus bairdii (Perissodactyla: Tapiridae) en La Reserva de La Biosfera "El Triunfo" (Polígono I), Chiapas, México. Acta Zoológica. Mexicana. (nueva serie), 20(1):1-21. 
Long, A. y M. Heath. 1991. Flora of the El Triunfo Biosphere Reserve, Chiapas, Mexico: A Preliminary Floristic Inventory and the Plant Communities of Polygon I. Anales del Instituto de Biología. Universidad Nacional Autónoma de México. Serie Botánica, 62:133-172.

Mandujano, S. 1991. Notas sobre el pecarí de collar en el bosque tropical caducifolio de Chamela, Jalisco. Pp.222 - 228, en: Memorias IX Simposio sobre Fauna Silvestre. Facultad de Medicina Veterinaria y Zootecnia, UNAM, México, D.F.

March, I. J. 1990. Evaluación del hábitat y situación actual del pécari de labios blancos Tayassu pecari en México. Tesis de Maestría. Programa Regional en Manejo de Vida Silvestre para Mesoamérica y el Caribe. Universidad Nacional Heredia, Costa Rica.

Meredíz, G. 1995. Abundancia, distribución y posibilidad de aprovechar sustentable del jabalí de collar (Tayassu tajacu) y otras especies faunísticas de la Zona Maya de Quintana Roo. Tesis Licenciatura. UNAM. México, D.F.

Naranjo, E. J. 2000. Estimaciones de Abundancia y Densidad en Poblaciones de Fauna Silvestre Tropical. Pp. 37-46, en: Manejo de Fauna Silvestre en Amazonía y Latinoamérica. (Cabrera, E. C. Mercolli, y R. Resquin, eds), Asunción, Paraguay.

Naranjo, E. J. 2002. Population ecology and conservation of ungulates in the Lacandon Forest, Mexico. Tesis Doctoral, Florida University,Gainesville.

Noss, A. y E. Cuellar. 2000. Índices de abundancia para fauna terrestre en el Chaco Boliviano: huellas en parcelas y en brechas barridas. Pp. 73-82, en: Manejo de Fauna Silvestre en Amazonía y Latinoamérica. (Cabrera, E. C. Mercolli, y R. Resquin, eds), Asunción, Paraguay.

Quijano, E. 1988. Distribución, abundancia y conocimiento tradicional de mamíferos silvestres: bases para la creación de un plan de manejo y aprovechamiento en Tres Reyes, Quintana Roo. Tesis de Licenciatura en Biología. UNAM, México, D.F.

Quinto, F. 1994. Estudio sobre la distribución y abundancia del jabalí de collar en Ejidos Forestales de Quintana Roo. Memorias del XII Simposio sobre Fauna Silvestr. Facultad de Medicina Veterinaria y Zootecnia, UNAM. México, D.F.

Piagram, J. J. 1980. Environmental implications of tourism development, Annals of Tourism Research.7: 554 - 583.

Polisar, J., R.M. McNab, H. Quigley, M. J. González y M. Cabrera. 1998. A preliminary assesment of the effects of subsistence hunting in the Maya Biosphere Reserve. Informe al WCS - Flores Peten Guatemala. Inédito.

Rzedowsky, J. 1991. Vegetación de México. Limusa. México, D.F.

Schaller, G. B. 1967. The deer and the tiger. University of Chicago Press, Chicago.

Sokal, R. R. y J.F. Rohlf. 1995. Biometry. Segunda edición. W.H. Freeman and Co., Salt Lake City,Utah.

Thomas, L., J.L. Laake, J.F. Derry, S.T. Buckland, D.L. Borchers, D.R. Anderson, K.P. Burnham, S. Strindberg, S.L. Hedley, M.L. Burt, F. Marques, J.H. Pollard, y R.M. Fewster. 1998. Distance 3.5. Research Unit for Wildlife Population Assessment, University of St. Andrews, Reino Unido.

Touval, J. 1993. Ecotourism and its Role in the Conservation of El Triunfo Biosphere Reserve, Chiapas, Mexico. Tesis de Maestría, University of Maryland at College Park. Maryland 
Townsend, W. 1996. La utilidad del monitoreo del uso de la cacería para la defensa de un territorio. Pp. 177 - 189, en: Manejo de Fauna con Comunidades Rurales. ( Campos, C., Ulloa, A. Rubio H, eds.), Bogotá, Colombia.

Wallace, R. B. y R. L. Painter. 2000. Conservación de pecaríes en la Amazonía Boliviana: biodiversidad vs viabilidad poblacional. Pp 263 - 271, en: Manejo de Fauna Silvestre en Amazonía y Latinoamérica. ( Cabrera, E. C. Mercolli, y R. Resquin, eds). Asunción, Paraguay. 